\title{
The application of "dysexecutive syndrome" measures across cultures: Performance and checklist assessment in neurologically healthy and traumatically brain-injured Hong Kong Chinese volunteers
}

\author{
RAYMOND C. K. CHAN ${ }^{1}$ AND TOM MANLY ${ }^{2}$ \\ ${ }^{1}$ Department of Psychiatry, The University of Hong Kong \\ ${ }^{2}$ MRC Cognition and Brain Sciences Unit, Cambridge UK
}

(Received July 26, 2000; Revised September 20, 2001; Accepted October 9, 2001)

\begin{abstract}
Deficits in planning, self-regulation and attention are a relatively common consequence of traumatic brain injury (TBI). Such "dysexecutive" deficits tend to be most exposed in complex, real world situations. Consequently, clinicians often have to rely on interviews, questionnaires and observation in their assessments. While there is little doubt that dysexecutive symptoms occur across different cultures, the expression of those symptoms, the way in which they are experienced by others, and the propensity of friends/relatives to report negative features may vary considerably. The cross-cultural use of standardized checklists and measures that have predominantly been studied with English speaking, Western groups therefore requires empirical support. Here a group of 68 healthy Chinese speaking volunteers were asked to complete translations of 2 UK developed questionnaires (the Dysexecutive Questionnaire and Cognitive Failures Questionnaire) measures and to perform 2 "executive" tasks (The Six Elements Test and the Tower of Hanoi). Their self ratings and the ratings of close relatives were very close to those seen in the original UK standardization samples - as was their performance on the 2 tasks. Accordingly, the conditions for assessing their clinical sensitivity were met. Comparison between 30 Chinese patients with TBI and matched controls showed that both questionnaires and tests were sensitive to the deficits in this group.

(JINS, 2002, 8, 771-780.)
\end{abstract}

Keywords: Assessment, Dysexecutive syndromes, Brain injury, Cultures

\section{INTRODUCTION}

Damage to the frontal lobes can lead to a disastrous inability to cope in many real world situations. Patients may, for example, begin to make unrealistic decisions, get impulsively caught up in actions despite them being irrelevant to their goal or even contrary to their stated intentions. They may become highly distractible, insensitive to feedback and lose a great deal of subtlety in interpersonal relationships (Burgess \& Shallice, 1996; Lhermitte, 1985; Luria, 1966; Prigatano, 1991; Robertson et al., 1997; Schwartz, 1995; Shallice, 1988; Shallice \& Burgess, 1991; Stuss \& Benson,

Reprint requests to: Raymond C. K. Chan, Department of Psychiatry, The University of Hong Kong, Pokfulam Road, Hong Kong, China. E-mail: ckrchan@graduate.hku.hk
1986). While not invariably the case, such deficits can occur despite many cognitive functions appearing to be relatively intact on formal neuropsychological testing (Shallice, 1988; Shallice \& Burgess, 1991). This discrepancy has led many authors to propose that the frontal lobes exert an executive, coordinating role, orchestrating other capacities in order to achieve goals (Duncan, 1986; Fuster, 1989; Luria, 1966; Shallice, 1988).

It has been argued that in many standard tests, the clear instructional set and well defined goal, the strong cues about when to start and stop activity, and the direction of attention towards relevant information may do much to offset demands on an executive system. In contrast, real life brings many situations that are unpredictable, where the desired outcome may be ambiguous, and where individuals need be flexible in adjusting their behavior as events develop. 
Despite the inherent difficulties in capturing such complex deficits, controlled quantification remains a desirable goal for both clinicians and academics. A principal approach to the frontal lobe or dysexecutive syndrome has therefore been to develop tasks that maximally tax one or other hypothesized impairment. The widely used Wisconsin Card Sorting Test (Nelson, 1976; Heaton et al., 1981, 1993) for example, requires patients to infer a changing rule based on the tester's feedback and flexibly adapt their behavior accordingly. Tests of verbal fluency (e.g., Miller, 1984) require patients to generate a strategy for retrieval and avoid getting stuck in a perseverative "rut." While such tests can clearly be powerful in discriminating patients from control groups, the predictive value of such abstract tasks for everyday difficulties for any given individual has been questioned (Burgess, 1997; Burgess et al., 1998; Sbordone, 1996). It is certainly the case that patients may perform well on each of these and many other frontal measures and yet experience highly debilitating dysexecutive deficits in their day-to-day lives (Prigatano, 1991; Shallice \& Burgess, 1991).

A further difficulty lies in the apparent fractionation of the dysexecutive syndrome (Burgess, 1997; Burgess et al., 1998), meaning that a patient's performance on one frontal test may have little or no predictive value for how they may perform on another test, let alone in a complex real world situation. As we discuss further below, in addressing this problem there is an increasing emphasis on incorporating more complex, multifaceted and life-like challenges within performance measures, in other words tasks that will tap a number of executive domains at the same time (Schwartz et al., 1991; Shallice \& Burgess, 1991; Whyte et al., 1994; Wilson et al., 1996). For the clinician, however, behavior in real-life must always remain the crucial test. Checklists and questionnaires offer one route that, in principal, can distill observation of everyday difficulties into useable and reliable form. The study we describe here is principally concerned with whether such measures remain useful when applied in a very different culture from the one in which they were developed.

\section{Two Checklist Measures of Dysexecutive Behavior}

\section{The Dysexecutive Questionnaire (DEX; Wilson et al., 1996)}

In standardizing the Behavioural Assessment of the Dysexecutive Syndrome (BADS), a battery of executive performance tests, Wilson et al. (1996) developed a 20-item checklist of dysexecutive symptoms-the Dysexecutive Questionnaire (the DEX). The DEX asks individuals to rate the frequency of occurrence of certain dysexecutive characteristics, for example, in abstract thinking, impulsivity, confabulation and planning problems. Items included, I sometimes act without thinking, doing the first thing that comes to mind and I am unconcerned about how I should behave in certain situations. Parallel versions of the questionnaire were developed, one to be completed by the patient and one by a close friend or relative about the patient.

A Varimax rotated factor analysis of the responses of relatives of brain injured patients to the DEX revealed three rather independent cognitive factors (termed Inhibition, Intentionality and Executive Memory) and two smaller factors related to emotional experience (Burgess et al., 1998). Separate comparison of the relationships between the subgroups of items best reflecting the factors and test performance revealed a complex pattern. For example, the Inhibition factor was significantly related to test performance across a wide range of both executive and nonexecutive tests, consistent with either the common demands of many tests for inhibition, or with this factor reflecting a more general level of cognitive impairment. The second and third factors, Intentionality and Executive Memory were more specific, the former being only related to performance on the Six Elements test (see below) with the later being the only factor to show good relationships to memory test performance.

It should be noted that the above relationships were only apparent for the relative report versions of the DEX. Although the patients' self-reports revealed higher levels of daily life problems than the self-reports of neurologically healthy controls, the predictive value of the patient reports for test performance was almost completely negligible (Burgess et al., 1998; Wilson et al., 1996). This highlights an inherent problem for self-report checklists in this area. Brain damage in general, and particularly features of the dysexecutive syndrome can act against good insight into current difficulties, and hence undermine the predictive value of self-report scales for the clinician (see Oddy et al., 1985; Prigatano, 1991; Stuss, 1991; Sunderland et al., 1983). Such apparent lack of awareness of one's own deficits or inappropriate behavior is not necessarily related to the extent of cognitive impairments on standard neuropsychological tasks (Prigatano, 1991), and has been theoretically linked to damage to higher-level "integration" circuits in prefrontal and temporal cortices-for example in linking memory or selfawareness to current behavior (Prigatano, 1991).

\section{The Cognitive Failures Questionnaire \\ (Broadbent et al., 1982)}

The occurrence of "absent-minded" lapses-where action deviates from intention-have been highly influential in views of normal executive function and dysexecutive consequences of brain injury (e.g., Norman \& Shallice, 1980; Shallice, 1988). Broadbent and colleagues developed a 25item checklist on the frequency with which such errors occur. Items on the Cognitive Failures Questionnaire (CFQ) include Do you read something and find you haven't been thinking about it and must read it again? and Do you find you accidentally throw away the thing you want and keep what you meant to throw away-as in the example of throwing away the matchbox and putting the used match in your 
pocket? Independent validation of the measure was established through comparison with informant reports concerning healthy participants (Broadbent et al., 1982) —as well as relationships to frequency of accidents (Larson et al., 1997). Factor analysis of the CFQ on a population of 2,379 US citizens suggests that the majority of items load onto a common factor, with a small secondary factor largely relating to forgetting people's names (Larson et al., 1997). While the measure was not primarily developed as a neuropsychological tool, there is evidence of its sensitivity to traumatic brain injury, to the severity of that injury, and of its relationship to performance measures in brain injured groups (Robertson et al., 1997). As with the DEX, the results of Robertson et al.'s study showed the CFQ self-reports of brain injured volunteers to be less predictive of performance than those of their relatives.

\section{The Current Study}

Published studies of the dysexecutive syndrome have predominantly considered English speaking participants and, almost invariably, Western cultural groups. As a consequence, the majority of assessment measures and checklists, including the DEX and CFQ, are standardized and normed in English.

It seems reasonable to assume that similarities in the consequences of brain damage between different cultural groups on performance measures will far outweigh any differences. The subjective reports of symptoms, and the propensity to report these in others may, however, be more vulnerable to subtle or substantial linguistic or cultural differences.

We do not have the space to dwell on the potential cultural and linguistic differences between Hong Kong Chinese and UK samples here-particularly as both populations are themselves very diverse. The aim of this study is simply to establish whether the questionnaire measures appear to be sufficiently robust to what differences there are, thus justifying their clinical use with this community. To this end we examine the responses to a Cantonese translation of the DEX and CFQ questionnaires from 30 traumatically brain injured (TBI) patients and 68 neurologically healthy volunteers, together with the informant reports of relative on the measures. Given the potential problems with insight among the patient group, the primary aim was to establish whether the reports of relatives would significantly differ between the two groups. A secondary aim was to compare the questionnaire scores with broadly equivalent samples from the UK population. Finally in order to provide some estimate of at least one aspect of predictive validity of the translated measures, all participants were also asked to perform two performance based measures argued to tap aspects of executive function.

Shallice and Burgess (1991) developed a novel measure, the Six Elements Test, designed to emphasize strategy formation and self-determined behavioral flexibility. In the test, patients are presented with six separate tasks and asked to try and do at least some of each within the set time limit. The crucial aspect is that full completion of each task would take longer than the time available. The test therefore emphasizes a capacity to monitor one's own behavior against the main goal, to be aware to time and to switch tasks. A further rule is imposed constraining the order in which tasks are attempted. In their study, the test proved sensitive to a group of patients with frontal lesions from head injury who performed at above average levels on many standard tests (including WAIS intelligence scales, memory assessments and a number of frontal tasks) but who showed profound disorganization in everyday life. Subsequent support for the measure has come from its inclusion in the BADS where a group of 92 patients, predominantly with head injury, performed significantly below the level of the normative sample (Burgess et al., 1998; Wilson et al., 1996).

The Tower of Hanoi (Simon, 1975) and its derivatives are problem solving tasks that have been used extensively with brain damaged and other clinical groups (e.g., Arnett et al., 1997; Daum et al., 1995; Geol \& Grafman, 1995; Morris et al., 1997a, 1997b). In the task, participants are presented with disks arranged on a pegboard. They are asked to move the pegs from the starting state, in line with certain rules about movements, and to achieve a set end state. One feature that has been argued to best differentiate the performance of patients with frontal lobe lesions is where a move must be made ostensibly in opposition to the desired goal, for example, moving a disc from the peg it should occupy at the end of the task in order to allow the movement of another disk (Geol \& Grafman, 1995; Morris et al., 1997a).

In this study our aim was to establish whether the questionnaires would be useful for a Cantonese-speaking Chinese Hong Kong population. We tested the following hypotheses:

1. If the DEX and CFQ measures are robust to cultural and linguistic differences between the UK and Hong Kong populations and the translations are adequate, similar score levels should be seen in neurologically healthy samples from each location.

2. Given (1), the DEX and CFQ relatives' reports should prove sensitive to the presence of brain injury in the person being rated.

3. The brain injured participants should perform significantly more poorly than a matched control group on two translated executive performance measures: the Six Elements and the Tower of Hanoi.

4. The DEX and CFQ reports of relatives should have some predictive value for scores on the two performance tests (and, of course, vice versa), whereas the reports of the patients, in line with previous studies, should show a weaker or nonsignificant relationship. 


\section{METHODS}

\section{Research Participants}

\section{Neurologically healthy participants}

Sixty-eight participants from the healthy population were recruited via advertisements in universities and hospitals in Hong Kong. They all met the inclusion criteria of (1) having no history of head injury or other neurological or mental illness, (2) having no history of drug or alcohol problem, (3) having no visual and hearing difficulties likely to interfere with performance on the tests. The group of 34 women and 34 men was of mean age 37.5 years $(S D=7.79)$. The majority of the group had been resident in Hong Kong since birth ( $M$ years of residency $36.9, S D=8.61)$. All were of Chinese origin and spoke Cantonese as a first language. The mean number of years spent in full time education was $10.96(S D=2.78)$.

\section{Patients with TBI}

Patients with mild-to-moderate TBI, as defined by Teasdale and Jennett's system (Teasdale \& Jennett, 1974), were recruited from neurosurgical outpatient clinics in Hong Kong. The following inclusion criteria were used:

1. Patients between the ages of 20 and 55 who were at least 3 months post injury.

2. A Glasgow Coma Scale (GCS; Teasdale \& Jennett, 1974) score of at or above 8 within the first $24 \mathrm{hr}$ following the injury.

3. A period of unconsciousness or post-traumatic amnesia (PTA) not greater than 1 day.

4. No previous history of head injury, neurological or psychiatric illness.

5. No previous history of alcohol or drug abuse.

6. No speech, motor or perceptual deficit likely to interfere with completion of the tasks.

GCS, PTA duration and medical history were as reported by the neurologist with primary care of the patient. Thirty patients meeting the inclusion criteria gave informed consent to take part in the study. The 23 men and 7 women were of mean age 38.07 years $(S D=6.78)$. The patients median GCS score at the time of admission was 15 (range = $8-15)$. The median duration of loss of consciousness and post-traumatic amnesia following injury were both zero days (range $=0-1$ day).

Half of the group had no detectable abnormalities on CT scan-although this cannot be taken as ruling out functional damage. Thirty-three percent had clear scan evidence of frontal lobe damage, $10 \%$ had evidence of damage to the temporal lobes and $6.7 \%$ had evidence of damage to multiple regions.

At assessment the group was a mean of 3.45 months $(S D=38.16, M D N=15$ months $)$ post injury. Half of the group had returned to some form of work and only 6 of the patients were involved in legal cases relating to their injuries at the time of assessment. The hospital ethics board approved the recruitment and procedure. All patients gave written informed consent in accordance with the Declaration of Helsinki.

\section{Measures}

\section{The Dysexecutive Questionnaires}

(DEX; Wilson et al., 1996)

Both the self-report and informant-report versions of the DEX comprise 20 items describing aspects of the dysexecutive syndrome. Responses are given to each by endorsing one of a 5-point scale on the frequency with which that problem occurs [from never (zero) to very often (4)]. The items of the informant report version directly parallel those of the self-report version; for example, self-report: I act without thinking, doing the first thing that comes to mind, or informant report: [Name] acts without thinking, doing the first thing that comes to mind.

The initial DEX validation samples were large groups of brain-injured patients and healthy volunteers from the UK population (Wilson et al., 1996). The Cantonese translations were performed by the main author of this study (Chan, 2001). ${ }^{1}$ As our own check, the Cantonese versions of the measures were independently back-translated by another Cantonese speaker with any differences in key content areas identified used to revise the measures.

\section{The Cognitive Failures Questionnaires (CFQ; Broadbent et al., 1982)}

The self-report version and the informant-version of the CFQ were devised to examine the incidence and pattern of everyday action slips and cognitive errors. The self-report version has 25 items while the informant-report has eight items. The eight items of the informant-report CFQ were selected by Broadbent et al. (1982) to describe overt errors and traits that could be observed by others. The items do not directly parallel those of the self-report version.

Responses are given on a 5-point frequency scale [from never (zero) to very often (4)]. The score is the total for all of the items (maximum for self-report $=100$, informant report $=40)$.

\footnotetext{
${ }^{1}$ The DEX, the Six Elements and the Tower of Hanoi used in this study are copyright protected. The first author is pleased to accept correspondence from individuals wishing to use the translations given necessary permissions from the publishers of these measures. The first author is happy to accept enquiries about the CFQ translation as the original measure is in the public domain.
} 
The Modified Six Elements Test (Wilson et al., 1996)

The modification of Shallice and Burgess's (1991) original Six Elements task used in this study is part of the Behavioural Assessment of the Dysexecutive Syndrome Battery (Wilson et al., 1996). In the task participants are given four stimulus books, a tape player, a timer, blank paper, a pen, and a card showing the rules and aims of the test. They are told that the test requires them to perform three basic tasks, each with two halves (Parts A and B). One component task is to write down the names of items depicted in two stimulus books. Pictures from the first book form Part A of the task and pictures in the second book form Part B. The second task is to write down the solution to simple arithmetic problems presented in Books 3 and 4 (Book $3=$ Part A, Book $4=$ Part B). The final task is to dictate two specified episodic memory events into the tape recorder; a description of a holiday (Part A) and a memorable event (Part B).

Participants are asked to try to do some of each task during the $10 \mathrm{~min}$ of the test. They are told that they will not be able to fully complete all of the tasks within this time. The only rule that governs the order in which the tasks are presented in that parts A and B of any of the core tasks (e.g., naming pictures from Book 1 and Book 2) cannot be attempted successively.

The BADS Six Elements profile score (out of a maximum of 4) is calculated from the number of tasks attempted, the allocation of time to the tasks and the number of times that the rule is broken. In addition, individual parameters including the number of rule breaks, or the deviation in time allocation from an optimal level can be scored. This version of the Six Elements test, which has high interrater reliability, was found to be the most sensitive to brain injury of all of the subtests of the BADS (Burgess et al., 1998; Wilson et al., 1996).

\section{The Tower of Hanoi (Humes et al., 1997).}

In this task participants are presented with a three-peg board and four wooden disks of differing size. The task is to transfer the disks from a set starting position to a set final position in the minimum number of moves. This test comprised 12 items, with points being assigned based on the number of trials required for two consecutive solutions ( 6 points for Trials 1 and 2; 5 points for Trials 2 and 3; 4 points for Trials 3 and $4 ; 3$ points for Trials 4 and 5;2 points for Trials 5 and 6; and zero points if no consecutive correct trials were achieved). Three other scores were calculated based on the time from the start of the trial to the first action (initiation time), the total time for the participant to complete each problem (execution time), and the total number of rulebreaks within the task. Psychometric properties of this version of the task are given in Humes et al. (1997).

Both Six Elements and Tower of Hanoi tasks were translated from published versions by the main author of this study, the process checked through independent back-translation.

\section{Procedure}

Both control and patient volunteers were tested individually within a quiet office. The participants were asked to complete the DEX and CFQ, followed by the two performance measures within a fixed sequence. The total duration of testing procedures was approximately $1 \mathrm{hr}$.

Informant-report versions of the questionnaires were completed either in a separate room or taken away and subsequently returned by relatives accompanying the participants to the session. The only stipulation was that the questionnaires be completed by somebody who knew the patient well and who, ideally, lived in the same home.

\section{RESULTS}

\section{Performance From Neurologically Healthy Participants}

\section{Questionnaire measures}

All of the neurologically healthy participants completed the self-report version of the DEX. Informant report versions were available for 62 participants. The mean selfreport DEX score was $21.79(S D=8.96$; range $=4-45)$, with the mean for the informant-report versions being 20.39 $(S D=10.38$; range $=4-48)$. Both scores fall at approximately the same level as a UK sample of 216 neurologically healthy volunteers reported in Wilson et al. (1996) where the self-report mean was $20.99(S D=9.63)$ and the informant-report mean was 17.08 ( $S D=11.81$; J.J. Evans, personal communication). The frequency of report on individual items was not available from the original UK sample.

In this sample, scores on the informant-report version of the DEX were significantly correlated with those on the self-report version (Pearson's $r=.37, p<.01$ )

The mean self-reported score on the CFQ was 33.9 ( $S D=$ 10.4 ; range $=10-53$ ). This is strikingly similar to results from the largest published group study of CFQ self-reports based on the responses of 2379 US Navy recruits (Larson et al., 1997) where the mean lay at $33.6(S D=12.8)$.

The mean informant-reported score on the eight-item version of the CFQ was $10.89(S D=4.02)$. The results for the Hong Kong sample were compared with those of a recent UK sample of 55 neurologically healthy people upon whom informant CFQ reports were available. The sample comprised 17 men and 35 women of mean age 34.1 years $(S D=$ 9.95), who formed part of the control group previously reported in Robertson et al. (1997). There was no significant difference in the overall scores $[t(115)=0.43, p=.67]$ nor on any of the mean scores for the items (Item 1: $t=0.50$, $p=.62$; Item $2: t=-0.32, p=.75$; Item $3: t=0.85, p=$ .40 ; Item $5: t=1.53, p=.13$; Item $6: t=0.69, p=.49$; Item 7: $t=0.67, p=.50$; Item $8: t=1.25, p=.21)$ with the exception of Item 4 (Has $x$ been busy thinking about his / her own affairs and so not noticing what is going on around him/her?) where the UK sample had a modestly higher 
propensity to rate this as being a more frequent occurrence $(t=-2.56, p<.05)$. This difference would not however survive correction for multiple comparisons.

As with the original UK sample (Broadbent et al., 1982) there was a significant correlation between self-ratings and the independent ratings of a friend or relative on the CFQ $(n=64$; Pearson's $r=.50, p<.001)$.

Scores on the CFQ were significantly related to scores on the DEX, strikingly so in the case of the informant reports (CFQ-self-DEX-self: $r=.43, p<.01$; CFQ-other-DEXother: $r=.71, p<.001)$.

In summary, the ratings of the participants accorded closely with those of their relatives, and both measures appear to attract very similar levels of responses from neurologically healthy Hong Kong participants to those seen in previous healthy US and UK samples.

\section{Performance on the Six Elements Test}

The test was scored according to the BADS criteria yielding a profile score with a maximum of 4 . The volunteers scored a mean of $3.58(S D=0.56$; range $=2-4)$. Performance was therefore approximately equivalent to that seen in 216 UK neurologically healthy participants $(M=3.52$, $S D=0.80)$ presented in Wilson et al. (1996).

\section{Performance on the Tower of Hanoi}

The Tower of Hanoi, when scored according to Humes et al.'s (1997) formula, produced a mean total score of 63.18 ( $S D=$ 6.75). This is somewhat above - although within the range of - the mean for a group of younger participants reported by Humes et al. $(M=55.6, S D=11.42)$.

\section{Age and education effects}

Educational level was significantly related to the age of the participants, the younger volunteers tending to have spent longer in full-time study $(r=-0.30, p<.05)$. Neither selfnor informant-report versions of the questionnaires were significantly related to the age or educational level of the participants. If educational level is taken as an approximate estimate of IQ, this result is consistent with the low relationships observed between the CFQ and general ability estimates in two UK samples (Broadbent et al., 1982; Robertson et al., 1997). Relationships between the DEX questionnaires and estimates of premorbid IQ also failed to reach statistical significance in the sample described by Wilson et al. (1996).

Correlations between test performance and age/education were in general modest. Education was related to the Six Elements Test profile score (Pearson's $r=.29, p<.05$ ) but not to individual components within the task such as the number of rule breaks (Pearson's $r=-.07, p=.58$ ). Neither age nor education were significantly related to the time spent in planning or executing Tower of Hanoi moves, nor to the total score in this sample (Pearson's $r=.25, r=.07$, $r=.11$, respectively).

\section{Sensitivity to Brain Injury}

\section{Questionnaire measures}

There are reasonable grounds in the results presented above to suggest that both rating scales and performance measures are provoking similar results in 68 Hong Kong Chinese volunteers to those seen in neurologically healthy English speaking groups. This suggests that the translation of items and instructions is adequate, and that cultural factors are not generally influencing either self- or informantrating scale use. The conditions for evaluating the sensitivity of the measures to traumatic brain injury are therefore met.

Taken as a group, the healthy control volunteers were well matched in age with the patient group $[F(1,96)=$ $.009, p=.92]$. However the control group had significantly more years of education $[F(1,96)=8.08, p<.01]$ and significantly differed in their sex distribution $\left(\chi^{2}=8.29\right.$, $p<.01)$. As there were too many women in the control group and the education level of the group was too high, our strategy to form a matched sample was first to remove data from 25 women in order of educational level, taking out the highest first. This led to a group that was matched on sex, but still not on educational level. Consequently data from 7 men with the highest levels of education were removed. The resulting groups were well matched on age $[M$ control age $=39.39$ years, $S D=7.99 ; M$ patient age $=$ 37.30 years, $S D=9.02 ; F(1,64)=0.99, p=.32]$, education [control $M=9.08, S D=1.38$; patient $M=9.10, S D=$ $3.40 ; F(1,64)=0.001, p=.98]$ and sex distribution $\left(\chi^{2}=\right.$ $.02, p=.88)$.

\section{Comparisons of executive performance between patients with TBI and normal controls}

The performance of the control and patient groups was compared on key variables from the questionnaire and test measures using $t$ tests. Informant reports on 28 of the patients were completed by spouses, the remaining 2 by siblings. In each case the informant shared the home of the patient. The group means, standard deviations, $t$ and (two-tailed) $p$ values are presented in Table 1.

As shown in Table 1, the self-report scores on both the DEX and CFQ measures were significantly greater for the patients than for the neurologically healthy participants, a result that is consistent with some awareness among patients that they do experience difficulties in these areas. However, the CFQ and DEX ratings of informants appear to differentiate much more strongly between the groups. As the DEX self and other scores are based on directly equivalent parallel items, this was examined in a repeated measures ANOVA with version (self vs. other) and group status (patient vs. control) as the between-subjects factor. The significant interaction term $[F(1,62)=5.15, p<.05]$ confirms that status exerts a significant influence over the discrepancy between self and other scores. While, due to the nonparallel items of the CFQ self and other versions a similar analysis is not appropriate, the capacity of the two 
Table 1. Comparisons between 30 TBI patients and an age, sex, and educational level-matched control group

\begin{tabular}{|c|c|c|c|c|c|c|}
\hline \multirow[b]{2}{*}{ Measure } & \multicolumn{2}{|c|}{$\begin{array}{l}\text { Control group } \\
\quad N=36\end{array}$} & \multicolumn{2}{|c|}{$\begin{array}{l}\text { Patient group } \\
\qquad N=30\end{array}$} & \multirow[b]{2}{*}{$t$} & \multirow[b]{2}{*}{$p$} \\
\hline & $M$ & $(S D)$ & $M$ & $(S D)$ & & \\
\hline DEX self-report & 22.47 & (9.19) & 32.57 & $(16.21)$ & -3.18 & $<.01$ \\
\hline DEX informant report & 21.74 & $(10.23)$ & 38.50 & $(15.21)$ & -5.23 & $<.001$ \\
\hline CFQ self-report & 32.19 & $(11.15)$ & 46.17 & (18.98) & -3.72 & $<.001$ \\
\hline CFQ other report & 10.53 & $(4.19)$ & 17.93 & $(4.48)$ & -6.77 & $<.001$ \\
\hline 6 Elements (profile score) & 3.50 & $(0.56)$ & 2.73 & $(1.01)$ & -3.79 & $<.001$ \\
\hline 6 Elements (rule breaks) & 0.29 & $(0.63)$ & 0.50 & $(0.73)$ & -1.21 & .23 \\
\hline TOH (total score) & 64.09 & $(6.41)$ & 60.5 & $(10.24)$ & 1.67 & .10 \\
\hline TOH (planning time/s) & 3.90 & $(2.02)$ & 7.29 & $(6.46)$ & -2.82 & $<.01$ \\
\hline TOH (Execution time/s) & 27.20 & $(8.12)$ & 42.59 & $(17.39)$ & -4.51 & $<.001$ \\
\hline
\end{tabular}

Note. $p$ values are for two-tailed tests.

versions to differentiate between patient and control groups was examined using a discriminant function analysis. This reveals that the odds ratio of the CFQ-other report (a measure reflecting how likely an individual is to be correctly classified to group according to their score) was significantly greater than for the CFQ-self version $[t(57)=3.14$, $p<.01]$.

Despite the relatively narrow range offered by the Six Elements Test profile score, the patients scored significantly below the age and educational level matched control group. The lack of a difference in the number of rule breaks (the rule constraining the order in which tasks should be attempted) suggests that the primary deficit in this group was getting caught up in one task to the detriment of the overall goal. This is confirmed by subsequent analysis of the number of tasks attempted (control $M=5.68, S D=.68$; patient $M=4.47, S D=1.48 ; t=4.28, p<.001$ ).

The total score on the Tower of Hanoi did not significantly differ between the groups. However, two timing scores, the amount of time spent in planning and the amount of time spent in executing moves was significantly greater among the patient group.

\section{The predictive value of the questionnaires for test performance}

The group differences on both questionnaire and test measures suggest that predictive relationships between the two would be apparent within the sample as a whole. However, such cross-group correlations may be mediated by general factors of impairment rather than being specifically predictive. Accordingly, the correlations within only the patient group (where variance was higher) were examined.

Patient's self reports on the CFQ were not significantly related to performance on either the Six Elements Test (total score Pearson's $r=.006, p=.97$ ) or the Tower of Hanoi (total score Pearson's $r=-0.35, p=.06$ ), although the later approached significance.
Both other-report versions of the questionnaires were predictive of performance on the Tower of Hanoi task (DEX Pearson's $r=-0.49, p<.01$; CFQ Pearson's $r=-.52$, $p<.01)$. A stepwise regression entering the questionnaire variable (CFQ-other) with the highest correlation with the $\mathrm{TOH}$ task first, revealed that the addition of the DEX-other questionnaire added nonsignificantly to the predictive power of the equation [overall equation $F(1,27)=10.21, p<.01$; $t(\mathrm{CFQ}-$ other $)=-3.19, p<.01, t(\mathrm{DEX}-$ other $)=-.89$, $p=0.39$ ], suggesting that whatever is common to both measures (reflected in their own high intercorrelations) is mediating the relationship with performance on this task.

Neither of the informant questionnaire scores were significantly related to the Six Elements test. However, the informant-report DEX, as discussed, is comprised of three somewhat distinct cognitive factors, Inhibition, Intentionality, and Executive Memory (Burgess et al., 1998). In a previous study with a UK sample, only the Intentionality factor of the DEX was related to Six Elements performance (Burgess et al., 1998). To investigate whether a similar relationship might pertain here, the items of the informant report version were summed into the three factors. Although the factors were strongly intercorrelated (Factor 1:Factor 2: Pearson's $r=.67, p<.001$; Factor 1:Factor 3: Pearson's $r=.75, p<.001$; Factor 2:Factor 3: Pearson's $r=.56, p<.01)$ they showed somewhat different predictive values for the performance tasks. Factor 1 was significantly predictive of Tower of Hanoi (total score: Pearson's $r=-.56, p<.01]$ but not of Six Elements performance (total score: Pearson's $r=-.28, p=.12$ ). In contrast, Factor 2, Intentionality, was significantly related to Six Elements (total score: Pearson's $r=-.38, p<.05$ ). Its relationship to the Tower of Hanoi approached statistical significance (Pearson's $r=-0.35, p=.06$ ). Factor 3 was related only to the Tower of Hanoi (Pearson's $r=-.56$, $p<.01$; Six Elements: $-.05, p=.79$ ).

It has previously been suggested that the difference between the DEX-self and the DEX-other scores, where the 
items are direct parallels, may form a useful index of "insight" (Burgess et al., 1998). The relationship between insight and cognitive performance, however, is complex, as some authors (e.g., Prigatano, 1991) have suggested that, in that there may be somewhat separable neural bases, the two may dissociate. Here we examined this by subtracting the patients' DEX-self scores from those of their relatives. Interestingly, while the results presented above showed that the both DEX-self and DEX-other total scores formed nonsignificant correlates of the Six Elements task, the relationship between this derived difference measure and the Six Elements reached statistical significance (Pearson's $r$ DEXdifference score: Six Elements Test total score $=-.45, p<$ $.05)$. This index was not, however, significantly related to the Tower of Hanoi task (Pearson's $r$ DEX-difference: Tower of Hanoi $=-.19, p=.30)$. As the items of the CFQ are not parallel between the versions, this measure does not lend itself to such a direct 'insight' index.

\section{DISCUSSION}

It is possible that both linguistic and cultural differences could undermine the clinical value of rating scales standardized on UK English speaking samples for Hong Kong Chinese. In this study we have shown that two questionnaire measures of cognitive failures and dysexecutive symptoms elicit very similar levels of self-rating from Chinese healthy volunteers. Furthermore, the propensity of partners or relatives to report such symptoms does not appear to be markedly different from a UK sample, even down to similar mean values for item-by-item scores, at least on the CFQ where this information was available.

It is perhaps less surprising that two performance based measures, the Six Elements Test and the Tower of Hanoi, should elicit similar levels of performance in Chinese and UK samples. The results do suggest, however, that both the translations of complex behavioral descriptions within the questionnaires, and the somewhat subtle nuances of the test instructions were adequate.

These similarities do not tell us a great deal about the nature of any cultural differences between the Chinese Hong Kong and UK populations, simply that the questionnaires and measures are relatively robust to whatever differences there are. This is, however, an important precursor to investigating the sensitivity of the measures to traumatic brain injury within this population. In this study we examined a group with predominantly mild or moderate head injuries. The results showed the following:

1. The self-reports of patients on the DEX and CFQ are increased relative to those of the age/sex/education matched neurologically healthy control group.

2. The difference between the patient and control groups was more strongly seen in the reports of relatives on the questionnaire measures. While this may reflect an exaggeration of deficits by the relatives, it is more plausible to argue - in line with a number of previous reports (Oddy et al., 1985; Prigatano, 1991; Robertson et al., 1997; Stuss, 1991; Sunderland et al., 1983; Wilson et al., 1996) - that this reflects relatively poor insight by patients into the nature and consequences of their own deficits.

3. Of the two performance measures examined, the Six Elements Test, proved the more sensitive to traumatic brain injury in this sample. Significant control-patient differences in speed of execution on the Tower of Hanoi problem solving task were, however, noted.

4. The self-reports of patients had no significant predictive value for test performance, a result that is again consistent with a degree of lack of insight in the patient group. Relative reports on the CFQ and DEX were significantly related to Tower of Hanoi performance. Breaking the DEX questionnaire down into previously established cognitive components revealed that the Inhibition and Executive Memory factors had predictive value for the Tower of Hanoi task, while the Intentionality factor was related to the Six Elements test. Previous research with a UK sample has shown a similar divergence in predictive value (Burgess et al., 1998).

5. Previously it has been suggested that the difference between the DEX-other and DEX-self score could form a useful measure of insight (Burgess et al., 1998). In this sample, this difference score for patients was significantly correlated with Six Elements Test performance (poorer insight being related to poorer performance), and in this respect proved a stronger correlate than either DEX-self or DEX-other alone. The correlation was, however, relatively modest and no such relationship was seen with the Tower of Hanoi task. It is prudent therefore to interpret this result with some caution and as a possible starting point for further investigation.

Taken together the results support the general clinical applicability of these translated rating scales and tests to Chinese patient groups. In particular, the results emphasize and echo findings from previous studies on the importance of friend/relative reports in making predictions about performance on tests - and by extension-in everyday situations. They also suggest that the construction of the dysexecutive syndrome-as expressed in the items of the scales-has validity across cultures.

There are, of course, a number of limitations to the current study. Because both DEX and CFQ questionnaires were completed at the same session, carry-over effects may well have contributed to the size of the correlation observed between the measures - which, in turn, may also influence the degree of predictive validity of each measure for the performance tasks. This is, however, a complex issue to address in traumatic brain injury, as extending the interval between completion of one questionnaire or another may coincide with real changes within a patient's levels of impairment. Secondly, the questionnaire responses concern- 
ing neurologically healthy individuals give grounds to believe that any cultural differences between the samples are not exerting a strong influence. However, in respect of the head-injured group the question remains somewhat open. While we have information on severity, the number of cognitive/outcome measures used here was relatively small and we have limited grounds to claim equivalence to existing UK samples. Finally, we cannot extrapolate from these findings to the performance of these measures in other cultures. While they give grounds to believe that predominantly Western conceptualizations of the dysexecutive syndrome demonstrate cross-cultural validity, in each case these issues need to be empirically addressed.

\section{ACKNOWLEDGMENTS}

The authors would like to thank the Thames Valley Test Company and Dr. Marilyn Welsh for their kindness of permitting the translations of the DEX, Six Elements Test and Tower of Hanoi for research purposes. We are grateful to three anonymous reviewers for the careful consideration of the manuscript. This research was supported by the Sir Edward Youde Memorial Fund Fellowship and Erik Kvan Scholarship to the main author and by the UK Medical Research Council.

\section{REFERENCES}

Arnett, P.A., Rao, S.M., Grafman, J., Bernardin, L., Luchetta, T., Binder, J.R., \& Locbeck, L. (1997). Executive functions in multiple sclerosis: An analysis of temporal ordering, semantic encoding, and planning abilities. Neuropsychology, 11, 535-544.

Broadbent, D.B., Cooper, P.F., FitzGerald, P., \& Parkes, K.R. (1982). The Cognitive Failures Questionnaire (CFQ) and its correlates. British Journal of Clinical Psychology, 21, 1-16.

Burgess, P.W. (1997). Theory and methodology in executive function research. In P. Rabbitt (Ed.), Methodology of frontal and executive function (pp. 81-111). Hove, UK: Psychology Press.

Burgess, P.W., Alderman, N., Evans, J., Emslie, H., \& Wilson, B.A. (1998). The ecological validity of tests of executive function. Journal of the International Neuropsychological Society, 4, 547-558.

Burgess, P.W. \& Shallice, T. (1996). Bizarre responses, rule detection and frontal lobe lesions. Cortex, 32, 241-259.

Chan, R.C.K. (2001). Dysexecutive symptoms among non-clinical sample: A study with the use of Dysexecutive Questionnaire. British Journal of Psychology, 92, 551-565.

Daum, I., Schugens, M.M., Spieker, S., Poser, U., Schonle, P.W., \& Birbaumer, N. (1995). Memory and skill acquisition in Parkinson's disease and frontal lobe dysfunction. Cortex, 31, 413-432.

Duncan, J. (1986). Disorganisation of behaviour after frontal lobe damage. Cognitive Neuropsychology, 3, 271-290.

Fuster, J.M. (1989). The prefrontal cortex (2nd ed.). New York: Raven Press.

Geol, V. \& Grafman, J. (1995). Are the frontal lobes implicated in "planning" functions? Interpreting data from the Tower of Hanoi. Neuropsychologia, 33, 623-642.

Heaton, R.K., Chelune, G.J., Talley, J.L., Kay, G.G., \& Curtiss, G. (1993). Wisconsin Card Sorting Test manual. Odessa, FL: Psychological Assessment Resources.
Humes, G.E., Welsh, M.C., Retzlaff, P., \& Cookson, N. (1997). Towers of Hanoi and London: Reliability and validity of two executive function tasks. Assessment, 4, 249-257.

Larson, G.E., Alderton, D.L., Neideffer, M., \& Underhill, E. (1997). Further evidence on dimensionality and the Cognitive Failures Questionnaire. British Journal of Psychology, 88, 29-38.

Lhermitte, F. (1985). Human anatomy and the frontal lobes. Part II: Patient behavior in complex and social situations: The "environmental dependency syndrome." Annals of Neurology, 19, $335-343$.

Luria, A.R. (1966). Higher cortical functions in man. London: Tavistock.

Miller, E. (1984). Verbal fluency as a function of a measure of verbal intelligence and in relation to different types of pathology. British Journal of Clinical Psychology, 23, 52-57.

Morris, R.G., Miotto, E.C., Feigenbaum, J.D., Bullock, P., \& Polkey, C.E. (1997a). The effect of goal-subgoal conflict on planning ability after frontal- and temporal-lobe lesions in humans. Neuropsychologia, 35, 1147-1157.

Morris, R.G., Miotto, E.C., Feigenbaum, J.D., Bullock, P., \& Polkey, C.E. (1997b). Planning ability after frontal and temporal lobe lesions in humans: The effects of selection equivocation and working memory. Cognitive Neuropsychology, 14, 1007-1027.

Nelson, H. (1976). A modified card sorting test sensitive to frontal lobe deficits. Cortex, 12, 313-324.

Norman, D.A. \& Shallice, T. (1980). Attention to action: Willed and automatic control of behavior: Center for Human Information Processing.

Oddy, M., Coughlan, T., Tyerman, A., \& Jenkins, D. (1985). Social adjustment after closed head injury: A further follow-up seven years after injury. Journal of Neurology, Neurosurgery and Psychiatry, 48, 564-568.

Prigatano, G.P. (1991). Disturbances of self-awareness of deficit after traumatic brain injury. In G.P. Prigatano \& D.L. Schacter (Eds.), Awareness of deficit after brain injury: Clinical and theoretical issues (pp. 111-126). Oxford, UK: Oxford University Press.

Robertson, I.H., Manly, T., Andrade, J., Baddeley, B.T., \& Yiend, J. (1997). 'Oops!': Performance correlates of everyday attentional failures in traumatic brain injured and normal subjects. Neuropsychologia, 35, 747-758.

Sbordone, R.J. (1996). Ecological validity: Some critical issues for neuropsychologist. In R.J. Sbordone \& C.J. Long (Eds.), Ecological validity of neuropsychological testing (pp. 15-41). Delray Beach, FL: GR Press/St. Lucie Press.

Schwartz, M.F. (1995). Re-examining the role of executive functions in routine action production. Annals of the New York Academy of Sciences, 769, 321-335.

Schwartz, M.F., Reed, E.S., Montgomery, M., Palmer, C., \& Mayer, N.H. (1991). The quantitative description of action disorganization after brain damage: A case study. Cognitive Neuropsychology, 8, 381-414

Shallice, T. (1988). From neuropsychology to mental structure. Cambridge, UK: Cambridge University Press.

Shallice, T. \& Burgess, P. (1991). Deficit in strategy application following frontal lobe damage in man. Brain, 114, 727-741.

Simon, H.A. (1975). The functional equivalence of problem solving skills. Cognitive Psychology, 7, 268-288.

Stuss, D.T. (1991). Self, awareness and the frontal lobes: A neuropsychological perspective. In J. Strauss \& G.R. Goethals (Eds.), The self: Interdisciplinary approaches (pp. 255-278). New York: Springer. 
Stuss, D.T. \& Benson, D.F. (1986). The frontal lobes. New York: Raven Press.

Sunderland, A., Harris, J.E., \& Baddeley, A.D. (1983). Do laboratory tests predict everyday memory? A neuropsychological study. Journal of Verbal Learning and Verbal Behavior, 22, 341-357.

Teasdale, G. \& Jennett, B. Assessment of coma and impaired consciousness. Lancet, 2 (872), 81-84.
Whyte, J., Rose, T., Glenn, M.B., \& Gutowski, W. (1994). Quantification of attention-related behaviours in individuals with traumatic brain injury. American Journal of Physical Medicine and Rehabilitation, 73, 2-9.

Wilson, B.A., Alderman, N., Burgess, P.W., Emsley, H., \& Evans, J. (1996). Behavioural assessment of the dysexecutive syndrome. Bury St Edmonds, UK: Thames Valley Test Company. 